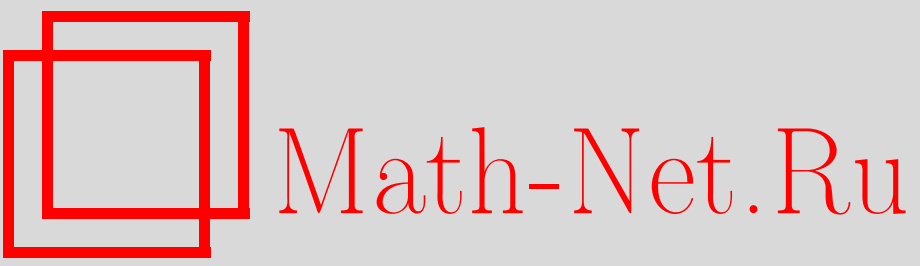

В. В. Духовый, М. П. Козловский, И. В. Пылюк, Расчет на микроскопическом уровне уравнения состояния трехмерной модели Изинга, ТМФ, 1996, том 107, номер 2, 288-306

DOI: https://doi.org/10.4213/tmf1156

Использование Общероссийского математического портала Math-Net.Ru подразумевает, что вы прочитали и согласны с пользовательским соглашением

http://www.mathnet.ru/rus/agreement

Параметры загрузки:

IP: 3.80 .181 .102

26 апреля 2023 г., 09:37:59 


\title{
РАСЧЕТ НА МИКРОСКОПИЧЕСКОМ УРОВНЕ УРАВНЕНИЯ СОСТОЯНИЯ ТРЕХМЕРНОЙ МОДЕЛИ ИЗИНГА
}

\begin{abstract}
Предложен новый метод расчета уравнения состояния трехмерной модели Изинга на простой кубической решетке с экспоненциально убывающим потенциалом взаимодействия. Полученное уравнение позволяет описать в критической области поведение параметра порядка как функцию температуры, внешнего поля и микроскопических параметров системы. Численное исследование температурной и полевой зависимости параметра порядка модели Изинга выполнено для случая соответствия параметров потенциала взаимодействию ближайших соседей.
\end{abstract}

\section{1. ПАРАМЕТР ПОРЯДКА ТРЕХМЕРНОЙ ОДНОКОМПОНЕНТНОЙ СИСТЕМЫ И РОЛЬ ДЛИННОВОЛНОВЫХ ФЛУКТУАЦИЙ}

Описание свойств системы при фазовом переходе может быть осушествлено с использованием основных принципов статистической механики. Способ размешения частиц и характер их взаимодействия позволяют найти термодинамические характеристики системы, например свободную энергию $F(V, T)$, и исследовать их зависимость от внешних параметров (температуры, поля). Указанный путь расчета позволяет сделать вывод о характере фазового перехода в данной физической системе. Проведенные ниже расчеты основываются на методе коллективных переменных (КП), обобшенном в $[1,2]$ на случай спиновых систем. Одно из преимушеств метода КП при описании фазовых переходов состоит в том, что среди множества его переменных $\left\{\rho_{\mathbf{k}}\right\}$ содержится переменная $\rho_{\mathbf{k}}$ (или их совокупность), относительно которой происходит смешение максимума функции распределения при переходе через критическую точку [2]. Индекс $\mathbf{k}^{*}$ этой переменной отвечает максимуму фурье-образа потенциала взаимодействия $\widetilde{\Phi}(k)$, а сама переменная связана с параметром порядка.

Для выполнения конкретных расчетов рассмотрим трехмерную модель Изинга с гамильтонианом

$$
H=-\frac{1}{2} \sum_{\mathbf{l}, \mathbf{j}} \Phi\left(r_{\mathbf{l}, \mathbf{j}}\right) \sigma_{\mathbf{l}} \sigma_{\mathbf{j}}-h \sum_{\mathbf{l}} \sigma_{\mathbf{l}} .
$$

В качестве потенциала взаимодействия $l$-й и $j$-й частиц, находящихся в узлах простой кубической решетки с периодом $c$, будем использовать экспоненциально убывающую функцию расстояния между ними

$$
\Phi\left(r_{\mathbf{1}, \mathbf{j}}\right)=A \exp \left(-r_{\mathbf{l}, \mathbf{j}} / b\right) .
$$


Здесь $A, b$ - постоянные ( $b$ - радиус эффективного действия потенциала). Фурье-образ потенциала (1.2) имеет вид

$$
\widetilde{\Phi}(k)=\widetilde{\Phi}(0)\left(1+b^{2} k^{2}\right)^{-2},
$$

где волновой вектор $\mathbf{k}$ меняется в пределах первой зоны Бриллюэна $(-B, B](B=\pi / c)$. При малых значениях волнового вектора для (1.3) справедлива параболическая аппроксимация. Для волновых векторов, близких к границе полузоны Бриллюэна, такая аппроксимация не имеет места. Здесь, однако, $\widetilde{\Phi}(k)$ является малой величиной по сравнению со значением $\widetilde{\Phi}(0)$. В дальнейших расчетах будем полагать, что вне сферы радиуса $B^{\prime}=(b \sqrt{2})^{-1}$ величина $\widetilde{\Phi}(k)$ равна нулю, а внутри нее справедлива параболическая аппроксимация:

$$
\widetilde{\Phi}(k)= \begin{cases}\widetilde{\Phi}(0)\left(1-2 b^{2} k^{2}\right), & k \leq B^{\prime}, \\ 0, & B^{\prime}<k \leq B .\end{cases}
$$

В случае выполнения соотношений

$$
\widetilde{\Phi}(0)=2 d J, \quad b=\frac{c}{2 \sqrt{d}},
$$

где $d$ - размерность пространства, такое приближение для $\widetilde{\Phi}(k)$ в области малых значений волновых векторов отвечает аналогичной аппроксимации фурье-образа потенциала взаимодействия ближайших соседей с постоянной взаимодействия $J$.

Фазовое пространство КП является естественным для описания явлений при фазовых переходах. Используя метод КП, в [1] был предложен новый способ вычисления статистической суммы модели Изинга вблизи точки фазового перехода, а в $[3,4]$ на его основе выполнен конкретный расчет термодинамических функций модели в критической области. В данной работе основное внимание обрашено на получение уравнения состояния с использованием формализма КП. КП $\rho_{\mathbf{k}}=\rho_{\mathbf{k}}^{c}-i \rho_{\mathbf{k}}^{s}$ для модели Изинга определяются с помошью соотношений типа аналитического функционала [2]:

$$
\hat{\rho}_{\mathbf{k}}=\int \rho_{\mathbf{k}} J(\rho-\hat{\rho})(d \rho)^{N}
$$

где оператор перехода от спиновых к КП является произведением $\delta$-фуннцций и может быть представлен в виде

$$
J(\rho-\hat{\rho})=\int(d \omega)^{N} \exp \left(2 i \pi \sum_{\mathbf{k}} \omega_{\mathbf{k}}\left(\rho_{\mathbf{k}}-\hat{\rho}_{\mathbf{k}}\right)\right),
$$

а операторы мод колебаний спиновой плотности выражаются через спиновые переменные

$$
\hat{\rho}_{\mathbf{k}}=1 / \sqrt{N} \sum_{\mathbf{l}} \sigma_{\mathbf{l}} \exp (i \mathbf{k} \mathbf{l}) .
$$

Вьполняя суммирование статистического оператора $\exp (-\beta H)$ по спиновым состояниям, для статистической суммы системы получаем

$$
Z=\int \exp \left(\frac{1}{2} \sum_{\mathbf{k}} \beta \Phi(k) \rho_{\mathbf{k}} \rho_{-\mathbf{k}}+\sqrt{N} \beta h \rho_{0}\right) J(\rho)(d \rho)^{N}
$$


где $\beta=(k T)^{-1}$. Выражение

$$
J(\rho)=\operatorname{Sp} J(\rho-\hat{\rho})
$$

представляет собой якобиан перехода от спиновых к КП. Явный вид для $J(\rho)$ был рассчитан в [1].

Статистическая сумма модели Изинга в представлении КП записывается следуюшим образом [2]:

$$
\begin{aligned}
Z= & 2^{N} 2^{\left(N^{\prime}-1\right) / 2} \exp \left(a_{0}^{\prime} N^{\prime}\right) \int \exp \left[-\frac{1}{2} \sum_{k \leq B^{\prime}} d^{\prime}(k) \rho_{\mathbf{k}} \rho_{-\mathbf{k}}-\right. \\
& \left.-\sum_{l \geq 2} \frac{1}{(2 l) !}\left(N^{\prime}\right)^{1-l} \sum_{\substack{\mathbf{k}_{1}, \ldots, \mathbf{k}_{2 l} \\
k_{i} \leq B^{\prime}}} a_{2 l}^{\prime} \rho_{\mathbf{k}_{1}} \ldots \rho_{\mathbf{k}_{2 l}} \delta_{\mathbf{k}_{1}+\cdots+\mathbf{k}_{2 l}}+\sqrt{N} \beta h \rho_{0}\right](d \rho)^{N^{\prime}} .
\end{aligned}
$$

Здесь $N^{\prime}$ - редуцированное число переменных $\left(N^{\prime}=N s_{0}^{-3}, s_{0}=\pi b \sqrt{2} / c\right)$. Для величины $d^{\prime}(k)$ имеем

$$
d^{\prime}(k)=a_{2}^{\prime}-\beta \widetilde{\Phi}(k) .
$$

Коэффициенты $a_{2 l}^{\prime}$ определяются согласно формулам $[3]^{1)}$

$$
\begin{aligned}
& a_{0}^{\prime}=\ln \left[(2 \pi)^{-1 / 2}\left(3 / \mathcal{M}_{4}^{\prime}\right)^{1 / 4} \exp \left(\left(y^{\prime}\right)^{2} / 4\right) U\left(0, y^{\prime}\right)\right], \\
& a_{2}^{\prime}=\left(3 / \mathcal{M}_{4}^{\prime}\right)^{1 / 2} U\left(y^{\prime}\right), \quad a_{4}^{\prime}=\left(3 / \mathcal{M}_{4}^{\prime}\right) \varphi\left(y^{\prime}\right), \ldots
\end{aligned}
$$

Здесь введены обозначения

$$
\mathcal{M}_{4}^{\prime}=2 s_{0}^{-3}, \quad y^{\prime}=2 \sqrt{3} \pi^{3 / 2}(b / c)^{3 / 2} .
$$

Как видно из приведенных выше выражений (1.12) и (1.13), начальные значения коэффициентов $a_{2 l}^{\prime}$ зависят от отношения радиуса действия потенциала $b$ к постоянной решетки $c$. В случае $b \gg c$ величина $y^{\prime}$ принимает большие значения и для функции параболического цилиндра Вебера $U\left(a, y^{\prime}\right)$ и их комбинаций

$$
\begin{aligned}
& U\left(y^{\prime}\right)=U\left(1, y^{\prime}\right) / U\left(0, y^{\prime}\right), \\
& \varphi\left(y^{\prime}\right)=3 U^{2}\left(y^{\prime}\right)+2 y^{\prime} U\left(y^{\prime}\right)-2
\end{aligned}
$$

можно воспользоваться разложениями в ряды по степеням малой величины $c / b$. В peзультате для коэффициентов $a_{2 l}^{\prime}$ получим

$$
\begin{aligned}
& a_{0}^{\prime}=-\frac{1}{2} \ln (2 \pi)-\frac{1}{4}(\pi \sqrt{2})^{-3}\left(\frac{c}{b}\right)^{3}+O\left(\frac{c}{b}\right)^{6} \\
& a_{2}^{\prime}=1-(\pi \sqrt{2})^{-3}\left(\frac{c}{b}\right)^{3}+O\left(\frac{c}{b}\right)^{6} \\
& a_{4}^{\prime}=2(\pi \sqrt{2})^{-3}\left(\frac{c}{b}\right)^{3}\left[1-7(\pi \sqrt{2})^{-3}\left(\frac{c}{b}\right)^{3}+O\left(\frac{c}{b}\right)^{6}\right]
\end{aligned}
$$

\footnotetext{
1) Приведенные ниже соотношения соответствуют приближению модели $\rho^{4}$. В случае использования более сложных моделей типа $\rho^{6}$ и выше получаем аналогичные выражения, в которых функции параболического цилиндра Вебера $U(a, t)$ переходят в более сложные специальные функции с большим числом аргументов (см [5]).
} 
Для достаточно больших значений $b$ находим

$$
a_{0}^{(G)}=\lim _{b / c \rightarrow \infty} a_{0}^{\prime}=-\frac{1}{2} \ln (2 \pi), \quad a_{2}^{(G)}=\lim _{b / c \rightarrow \infty} a_{2}^{\prime}=1,
$$

а все остальные $a_{2 l}^{\prime} \quad(l \geq 2)$ стремятся к нулю. Статистическая сумма $(1.10)$ в этом приближении принимает следующий вид:

$$
Z=2^{N-1 / 2} \frac{1}{\pi^{N^{\prime} / 2}} \int \exp \left[-\frac{1}{2} \sum_{k \leq B^{\prime}}(1-\beta \widetilde{\Phi}(k)) \rho_{\mathbf{k}} \rho_{-\mathbf{k}}+\sqrt{N} \beta h \rho_{0}\right](d \rho)^{N^{\prime}} .
$$

Температура фазового перехода $T_{c}^{(G)}$, соответствуюшая пределу $b / c \rightarrow \infty$, определяется соотношением

$$
\beta_{c}^{(G)} \widetilde{\Phi}(0)=1
$$

При температурах ниже $T_{c}^{(G)}$ выражение (1.16) приводит к нефизическим расходимостям свободной энергии и других характеристик модели, которые связаны с пренебрежением негауссовыми слагаемыми в (1.10). Последние являются малыми, однако оказывают принципиальное влияние на описание фазового перехода. Условие $b \gg c$ отвечает переходу к модели с дальнодействующим потенциалом взаимодействия. Он соответствует рассмотрению специфической модельной системы, которая не имеет реального физического аналога.

В дальнейшем будем считать, что радиус действия потенциала $b$ и постоянная решетки $c$ - соразмерные величины. В этом случае коэффициенты $a_{2 l}^{\prime}$ для всех значений $l \geq 0$ являются ненулевыми величинами и в показателе экспоненты подынтегрального выражения (1.10) необходимо принимать во внимание, кроме гауссовых, слагаемые, пропорциональные высшим степеням переменной $\rho_{\mathbf{k}}$.

Для проведения конкретных расчетов статистической суммы необходимо ограничиться в (1.10) конечным числом слагаемых. Как было показано в [2], базисным является четверное распределение, которое отвечает учету второй и четвертой степеней переменной $\rho_{\mathbf{k}}$ в (1.10). Однако получение количественных результатов для системы с гамильтонианом (1.1) требует дополнительного учета в выражении (1.10) слагаемых, пропорциональных $\rho_{\mathbf{k}_{1}} \ldots \rho_{\mathbf{k}_{6}}$. Подтверждением последнего являются результаты работы [5], где проводились расчеты по вычислению критического показателя корреляционной длины $\nu$ модели $\rho^{2 m}$ как функции величины $m$. В этой работе было установлено, что в процессе увеличения порядка модели зависимость $\nu$ от параметра ренормализационной группы $(\mathrm{P \Gamma}) s$ постепенно ослабляется и начиная с шестерной плотности меры $(m \geq 3)$ происходит незначительное изменение значения показателя $\nu$, имеющего тенденцию к насышению с ростом $m$. Таким образом, статистическая сумма будет рассматриваться нами в приближении модели $\rho^{6}$, т.е.

$$
\begin{aligned}
Z= & 2^{N} 2^{\left(N^{\prime}-1\right) / 2} \exp \left(a_{0}^{\prime} N^{\prime}\right) \int \exp \left[-\frac{1}{2} \sum_{k \leq B^{\prime}} d^{\prime}(k) \rho_{\mathbf{k}} \rho_{-\mathbf{k}}-\right. \\
& -\frac{1}{4 !} \frac{1}{N^{\prime}} \sum_{\substack{\mathbf{k}_{1}, \ldots, \mathbf{k}_{4} \\
k_{i} \leq B^{\prime}}} a_{4}^{\prime} \rho_{\mathbf{k}_{1}} \ldots \rho_{\mathbf{k}_{4}} \delta_{\mathbf{k}_{1}+\cdots+\mathbf{k}_{4}-} \\
& \left.-\frac{1}{6 !} \frac{1}{\left(N^{\prime}\right)^{2}} \sum_{\substack{\mathbf{k}_{1}, \ldots, \mathbf{k}_{6} \\
k_{i} \leq B^{\prime}}} a_{6}^{\prime} \rho_{\mathbf{k}_{1}} \ldots \rho_{\mathbf{k}_{6}} \delta_{\mathbf{k}_{1}+\cdots+\mathbf{k}_{6}}+\sqrt{N} \beta h \rho_{0}\right](d \rho)^{N^{\prime}} .
\end{aligned}
$$


Обшая идея получения уравнения состояния состоит в расчете наиболее вероятного значения $\rho_{0}$. В случае исследуемой модели переменная $\rho_{0}$ соответствует оператору $\hat{\rho}_{0}=$ $\frac{1}{N} \sum_{1} \sigma_{1}$, среднее значение которого связано с параметром порядка. Перейдем теперь к обсуждению условия, которое может быть использовано для получения уравнения состояния. Осушествим в (1.17) замену переменных

$$
\rho_{\mathbf{k}}=\rho_{\mathbf{k}}^{\prime}+\sqrt{N} \eta \delta_{\mathbf{k}}
$$

что эквивалентно выделению из переменной $\rho_{0}$ некоторой макроскопической величины. В результате такой замены из (1.17) находим

$$
Z=2^{N} 2^{\left(N^{\prime}-1\right) / 2} \exp \left(a_{0}^{\prime} N^{\prime}\right) \exp (-N E(\eta)) \int \omega(\rho, \eta)(d \rho)^{N^{\prime}}
$$

здесь

$$
E(\eta)=r \eta^{2}+u \eta^{4}+v \eta^{6}-\beta h \eta,
$$

где $r=\frac{1}{2}\left(a_{2}^{\prime}-\beta \widetilde{\Phi}(0)\right), u=\frac{a_{4}^{\prime}}{4 !} s_{0}^{3}, v=\frac{a_{6}^{\prime}}{6 !} s_{0}^{6}$, а выражение для $\omega(\rho, \eta)$ принимает вид (штрихи у $\rho_{0}$ и $\rho_{\mathbf{k}}$ опушены)

$$
\begin{gathered}
\omega(\rho, \eta)=\exp \left\{-\frac{1}{2} \sum_{k \leq B^{\prime}} \tilde{d}(k) \rho_{\mathbf{k}} \rho_{-\mathbf{k}}-\frac{\tilde{a}_{3}}{3 ! N^{\prime}} \sum_{k_{i} \leq B^{\prime}} \rho_{\mathbf{k}_{1}} \rho_{\mathbf{k}_{2}} \rho_{\mathbf{k}_{3}} \delta_{\mathbf{k}_{1}+\mathbf{k}_{2}+\mathbf{k}_{3}}-\right. \\
-\frac{\tilde{a}_{4}}{4 ! N^{\prime}} \sum_{k_{i} \leq B^{\prime}} \rho_{\mathbf{k}_{1}} \ldots \rho_{\mathbf{k}_{4}} \delta_{\mathbf{k}_{1}+\cdots+\mathbf{k}_{4}}-\frac{\tilde{a}_{5}}{5 !\left(N^{\prime}\right)^{2}} \sum_{k_{i} \leq B^{\prime}} \rho_{\mathbf{k}_{1}} \ldots \rho_{\mathbf{k}_{5}} \delta_{\mathbf{k}_{1}+\cdots+\mathbf{k}_{5}-} \\
\left.-\frac{\tilde{a}_{6}}{6 !\left(N^{\prime}\right)^{2}} \sum_{k_{i} \leq B^{\prime}} \rho_{\mathbf{k}_{1}} \ldots \rho_{\mathbf{k}_{6}} \delta_{\mathbf{k}_{1}+\cdots+\mathbf{k}_{6}}+\sqrt{N} \tilde{h}_{0}\right\} \\
\tilde{d}(k)=\tilde{a}_{2}-\beta \widetilde{\Phi}(k), \quad \tilde{a}_{2}=a_{2}^{\prime}+\frac{\eta^{2}}{2} s_{0}^{3}\left(a_{4}^{\prime}+\frac{a_{6}^{\prime} \eta^{2}}{12} s_{0}^{3}\right) \\
\tilde{a}_{3}=\sqrt{N} \eta\left(a_{4}^{\prime}+\frac{a_{6}^{\prime} \eta^{2}}{6} s_{0}^{3}\right), \quad \tilde{a}_{4}=a_{4}^{\prime}+\frac{a_{6}^{\prime} \eta^{2}}{2} s_{0}^{3} \\
\tilde{a}_{5}=a_{6}^{\prime} \sqrt{N} \eta, \quad \tilde{a}_{6}=a_{6}^{\prime}, \\
\tilde{h}=\beta h-d^{\prime}(0) \eta-\frac{\eta^{3}}{3 !} s_{0}^{3}\left(a_{4}^{\prime}+\frac{a_{6}^{\prime} \eta^{2}}{20} s_{0}^{3}\right) .
\end{gathered}
$$

Расчет величины $\eta$ осушествляется из известного уравнения

$$
\frac{\partial \widetilde{E}(\eta)}{\partial \eta}=0
$$

в котором

$$
\widetilde{E}(\eta)=E(\eta)+E_{1}(\eta)
$$

Здесь $E(\eta)$ задано в (1.20), а для $E_{1}(\eta)$ имеем

$$
E_{1}(\eta)=-\frac{1}{N} \ln Z_{\eta}
$$


где

$$
Z_{\eta}=\int \omega(\rho, \eta)(d \rho)^{N^{\prime}} .
$$

Среди решений этого нелинейного уравнения следует выбрать те, которые отвечают условию устойчивости системы. Вторая производная $\widetilde{E}(\eta)$ должна удовлетворять неравенству

$$
\frac{\partial^{2} \widetilde{E}(\eta)}{\partial \eta^{2}} \geq 0
$$

Знак равенства в (1.25) определяет границы устойчивости рассматриваемой фазы. Основная проблема при решении уравнения (1.21) состоит в корректном учете вклада от слагаемого $E_{1}(\eta)(1.23)$. Прежде чем приступить к ее решению, рассмотрим уравнение $(1.21)$ в простейшем приближении. Предположим, что $E_{1}(\eta)=$ const, a для $E(\eta)$ ограничимся выражением

$$
E(\eta)=r \eta^{2}+u \eta^{4}-x \eta
$$

здесь $r=r^{\prime}\left(T-T_{c}^{(G)}\right), r^{\prime}=a_{2}^{\prime} /\left(2 T_{c}^{(G)}\right), T_{c}^{(G)}=\widetilde{\Phi}(0) / a_{2}^{\prime}, x=\beta h$. Тогда согласно (1.21) параметр порядка (при $h=0$ ) определяется соотношением

$$
\eta= \pm\left(\frac{r^{\prime} T_{c}^{(G)}}{2 u}\right)^{1 / 2}\left|\tau_{0}\right|^{\beta}
$$

где

$$
\tau_{0}=\frac{T-T_{c}^{(G)}}{T_{c}^{(G)}} .
$$

Критический показатель $\beta$, который соответствует этому приближению, равен “классическому" значению $1 / 2$. При наличии внешнего поля $(h \neq 0)$ формула $(1.26)$ приводит к следуюшему уравнению состояния:

$$
2 r \eta+4 u \eta^{3}=x
$$

В случае $T=T_{c}^{(G)}$ параметр порядка

$$
\eta=(4 u)^{-1 / 3} x^{1 / \delta}
$$

где критический показатель $\delta$ принимает “классическое” значение $(\delta=3)$.

Значения критических показателей $\beta$ и $\delta$ не изменяются при включении в (1.26) слагаемого $v \eta^{6}$. Величина $E(\eta)$ в (1.19) является, по существу, разложением Ландау для свободной энергии по степеням параметра порядка [6]. Коэффициент возле $\eta^{2}$ пропорционален ${ }^{2)} T-T_{c}^{(G)}$, а коэффициенты возле высших степеней $\eta$ являются постоянными $[6,7]$. Отличие "классических" критических показателей от истинных (см., например, [8]) связано с пренебрежением зависимости от $\eta$ в выражении для $E_{1}(\eta)$, входящем в состав (1.22). Таким образом, можно сделать вывод, что использовать разложение Ландау (1.20) для построения уравнения состояния возможно только при условии, что величина (1.23) как функция $\eta$ не влияет сушественным образом на $\widetilde{E}(\eta)$ из $(1.22)$. Как будет

\footnotetext{
${ }^{2)}$ Пропорциональность $r$ к $T-T_{c}^{(G)}$ связана с пренебрежением зависимости от $\eta$ в $\omega(\rho, \eta)$.
} 
отмечено ниже, это справедливо вдали от точки фазового перехода, однако не имеет места в критической области. В этой области интегрирование по переменным $\rho_{\mathbf{k}}$ В $(1.24)$ приводит к существенному отличию выражений $E(\eta)$ и $\widetilde{E}(\eta)$.

Учет в (1.21) слагаемого $E_{1}(\eta)$ согласно (1.22)-(1.24) составляет один из возможных способов расчета параметра порядка. Основой этого способа является получение явного выражения $Z_{\eta}$ как функции $\eta$. Вид подынтегральной функции, входящей в (1.24), свидетельствует о довольно сложном пути реализации указанного способа. Второй способ заключается в том, что более простое исходное выражение из (1.17) интегрируется по всем переменным $\rho_{\mathbf{k}}$, кроме $\rho_{0}$, а вычисление параметра порядка $\eta$ связано с нахождением значения КП $\rho_{0}$, при котором имеет место экстремум подынтегрального выражения $[4,9]$

$$
Z=Z_{\mu} \int \exp E\left(\rho_{0}\right) d \rho_{0}
$$

Здесь $Z_{\mu}$ - не зависящая от параметра порядка часть статистической суммы. Выражение для $E\left(\rho_{0}\right)$ получается путем последовательного исключения из рассмотрения КП $\rho_{\mathbf{k}} \mathrm{c} k \neq 0$.

В настояшей работе предложен объединенный вариант первого и второго описанных выше способов расчета уравнения состояния. Сначала осушествляется интегрирование статистической суммы (1.17) по "несушественным" переменным $\rho_{\mathbf{k}}$ с $B_{m_{\tau}}<k \leq B^{\prime}$, где $B_{m_{\tau}}=B^{\prime} s^{-m_{\tau}}, m_{\tau}$ - номер слоя фазового пространства КП, после которого происходит выход системы из критического режима $(\mathrm{KP})^{3)}$. Это соответствует идеологии второго способа расчета. Затем, когда размеры эффективных спиновых блоков становятся порядка корреляционной длины системы $\xi$ (и при дальнейшем интегрировании превышали бы его), применяется первый способ получения уравнения состояния. Преимущество предложенного метода получения уравнения состоит в том, что в выражении для $\widetilde{E}(\eta)(1.22)$ величина $E_{1}(\eta)$ не изменяет зависимости $\widetilde{E}(\eta)$ от $\eta$, т.к. при рассмотрении достаточно больших по размерам эффективных спиновых блоков она стремится к постоянной величине. С другой стороны, если температура не близка к $T_{c}$, отпадает необходимость в предварительном исключении "несушественных" переменных $\rho_{\mathbf{k}}$, поскольку в этом случае корреляционная длина системы соразмерна с радиусом действия потенциала взаимодействия $(\xi \sim b \sim c)$. Здесь хорошо работает теория Ландау и выражение (1.20) вполне может быть использовано для построения уравнения состояния.

Следующий раздел посвяшен методу расчета уравнения состояния непосредственно в критической области.

\section{2. УРАВНЕНИЕ СОСТОЯНИЯ ОДНОКОМПОНЕНТНОЙ СПИНОВОЙ СИСТЕМЫ}

Рассмотрим статистическую сумму (1.17) изинговской системы. Ее вычисление осуществляется путем “послойного" интегрирования согласно методики, предложенной в $[1,2]$. При $T>T_{c}$ статсумма приобретает вид $[3,9]$

$$
Z=2^{N} 2^{\left(N_{m_{\tau}+1}-1\right) / 2} Q_{0} Q_{1} \ldots Q_{m_{\tau}}\left[Q\left(P_{m_{\tau}}\right)\right]^{N_{m_{\tau}+1}} \int \omega_{m_{\tau}+1}(\rho)(d \rho)^{N_{m_{\tau}+1}}
$$

\footnotetext{
3) Область КР характеризуется РГ-симметрией и соответствует наличию сильноскоррелированных спиновых блоков [2].
} 
Здесь $Q_{n}$-парциальная статистическая сумма $n$-й блочной структуры, $N_{m_{\tau}+1}=N^{\prime} \times$ $s^{-3\left(m_{\tau}+1\right)}$. В рамках модели $\rho^{6}$ эффективная плотность меры $\omega_{m_{\tau}+1}(\rho)$ представляется следуюшим образом:

$$
\begin{aligned}
\omega_{m_{\tau}+1}(\rho)= & \exp \left\{-\frac{1}{2} \sum_{k \leq B_{m_{\tau}+1}}\left(s^{-2\left(m_{\tau}+1\right)} r_{m_{\tau}+1}+2 \beta \widetilde{\Phi}(0) b^{2} k^{2}\right) \rho_{\mathbf{k}} \rho_{-\mathbf{k}}-\right. \\
& -\frac{u_{m_{\tau}+1}}{4 ! N^{\prime}} s^{-\left(m_{\tau}+1\right)} \sum_{k_{i} \leq B_{m_{\tau}+1}} \rho_{\mathbf{k}_{1} \ldots \rho_{\mathbf{k}_{4}} \delta_{\mathbf{k}_{1}+\cdots+\mathbf{k}_{4}}-} \\
& \left.-\frac{\omega_{m_{\tau}+1}}{6 !\left(N^{\prime}\right)^{2}} \sum_{k_{i} \leq B_{m_{\tau}+1}} \rho_{\mathbf{k}_{1}} \ldots \rho_{\mathbf{k}_{6}} \delta_{\mathbf{k}_{1}+\cdots+\mathbf{k}_{6}}+\sqrt{N} \beta h \rho_{0}\right\},
\end{aligned}
$$

a $Q\left(P_{m_{\tau}}\right)$ выражается через функции основных и промежуточных аргументов (см. $[9,10])$. Выполним в $(2.1)$ замену переменных

$$
\rho_{\mathbf{k}}=\rho_{\mathbf{k}}^{\prime}+\sqrt{N} \sigma \delta_{\mathbf{k}},
$$

а значение $\sigma$ определим из уравнения

$$
\frac{\partial E^{(1)}\left(\sigma, m_{\tau}+1\right)}{\partial \sigma}=0,
$$

где

$$
\begin{aligned}
E^{(1)}\left(\sigma, m_{\tau}+1\right)= & -N\left(\frac{1}{2} s^{-2\left(m_{\tau}+1\right)} r_{m_{\tau}+1} \sigma^{2}+\right. \\
& \left.+\frac{1}{4 !} s_{0}^{3} s^{-\left(m_{\tau}+1\right)} u_{m_{\tau}+1} \sigma^{4}+\frac{1}{6 !} s_{0}^{6} \omega_{m_{\tau}+1} \sigma^{6}-\beta h \sigma\right) .
\end{aligned}
$$

Следует отметить, что замена переменных (2.3) на первый взгляд кажется эквивалентной (1.18), где было получено выражение для разложения Ландау по степеням параметра порядка. Однако она является принципиально иной. Напомним, что выражение (1.20) будет правильно описывать критическое поведение системы только в том случае, когда величина $Z_{\eta}(1.24)$ содержит слабую зависимость от $\eta$ и не меняет принципиальным образом выражение для $\widetilde{E}(\eta)$. Такая ситуация имеет место тогда, когда $\omega(\rho, \eta)$ является гауссовой плотностью меры и предусматривает при интегрировании статсуммы разделение переменных. Поэтому замена переменных (2.3) осушествляется на $m_{\tau}+1$-м этапе интегрирования статистической суммы, поскольку распределение фаз флуктуаций для блочных структур при $n>m_{\tau}$ становится гауссовым [3]. Для получения решения (2.4) необходимо определить точку выхода $m_{\tau}$ системы из КР. Примем в качестве определения величины $m_{\tau}$ уравнение

$$
\frac{r_{m_{\tau}+1}-r^{(0)}}{r^{(0)}}=-\delta,
$$

где $\delta$ - некоторая постоянная $(0<\delta \leq 1)$. Вблизи фиксированной точки величины $r_{n}$, $u_{n}, \omega_{n}$ удовлетворяют формулам [10]

$$
\begin{aligned}
& r_{n}=r^{(0)}+c_{1} E_{1}^{n}+c_{2} \omega_{12}^{(0)}\left(u^{(0)}\right)^{-1 / 2} E_{2}^{n}+c_{3} \omega_{13}^{(0)}\left(u^{(0)}\right)^{-1} E_{3}^{n}, \\
& u_{n}=u^{(0)}+c_{1} \omega_{21}^{(0)}\left(u^{(0)}\right)^{1 / 2} E_{1}^{n}+c_{2} E_{2}^{n}+c_{3} \omega_{23}^{(0)}\left(u^{(0)}\right)^{-1 / 2} E_{3}^{n}, \\
& \omega_{n}=\omega^{(0)}+c_{1} \omega_{31}^{(0)} u^{(0)} E_{1}^{n}+c_{2} \omega_{32}^{(0)}\left(u^{(0)}\right)^{1 / 2} E_{2}^{n}+c_{3} E_{3}^{n} .
\end{aligned}
$$


Здесь $E_{l}$ - собственные значения матрицы $\mathcal{R}$ преобразования

$$
\left(\begin{array}{c}
r_{n+1}-r^{(0)} \\
u_{n+1}-u^{(0)} \\
\omega_{n+1}-\omega^{(0)}
\end{array}\right)=\mathcal{R}\left(\begin{array}{c}
r_{n}-r^{(0)} \\
u_{n}-u^{(0)} \\
\omega_{n}-\omega^{(0)}
\end{array}\right)
$$

Координаты фиксированной точки

$$
\begin{aligned}
r^{(0)} & =-f_{0} \beta \widetilde{\Phi}(0), \\
u^{(0)} & =\varphi_{0}(\beta \widetilde{\Phi}(0))^{2}, \\
\omega^{(0)} & =\psi_{0}(\beta \widetilde{\Phi}(0))^{3}
\end{aligned}
$$

характеризуются универсальными величинами, которые в приближении модели $\rho^{6}$ соответственно равны $f_{0}=0,6166, \varphi_{0}=0,6426, \psi_{0}=0,5178$.

Коэффициенты $c_{l}$ как функции температуры приведены в работе [9]. Выражения для не зависимых от температуры величин $\omega_{i l}^{(0)}$ содержатся в [10]. Используя явные решения (2.7) рекуррентных соотношений (PC) для величин $r_{n}, u_{n}, \omega_{n}$ и принимая во внимание тот факт, что $E_{2}^{m_{\tau}+1} \ll 1$ и $E_{3}^{m_{\tau}+1} \ll 1$, получаем уравнение для определения $m_{\tau}$ :

$$
c_{1} E_{1}^{m_{\tau}+1}=\delta f_{0} \beta \widetilde{\Phi}(0)
$$

Это уравнение можно обобщить, учитывая слагаемые, пропорциональные $E_{l}^{m_{\tau}+1}$ $(l \geq 2)$, что приведет к скейлинговым поправкам (см. [3]). Исходя из (2.9) для величины $m_{\tau}$ находим

$$
m_{\tau}=-\frac{\ln \tau}{\ln E_{1}}+m_{0}-1,
$$

где

$$
\tau=\left(T-T_{c}\right) / T_{c}, \quad m_{0}=\ln \left(\delta f_{0} /{\tilde{c_{1}}}^{(0)}\right) / \ln E_{1} .
$$

Для $\tilde{c}_{1}^{(0)}$ имеем [9]

$$
\tilde{c}_{1}^{(0)}=V_{1}\left[1-f_{0}+v_{12}^{(0)} \varphi_{0}^{1 / 2}+v_{13}^{(0)} \psi_{0} \varphi_{0}^{-1}+\frac{a_{4}^{\prime} v_{12}^{(0)} \varphi_{0}^{-1 / 2}}{\left(\beta_{c} \widetilde{\Phi}(0)\right)^{2}}+\frac{2 a_{6}^{\prime} v_{13}^{(0)} \varphi_{0}^{-1}}{\left(\beta_{c} \widetilde{\Phi}(0)\right)^{3}}\right],
$$

здесь

$$
\begin{aligned}
v_{12}^{(0)} & =\left(E_{1}-R_{11}-e_{0} R_{31}^{(0)}\right) / R_{21}^{(0)}, \quad v_{13}^{(0)}=e_{0}, \\
e_{0} & =\frac{\left(E_{1}-R_{11}\right) R_{23}^{(0)}+R_{21}^{(0)} R_{13}^{(0)}}{\left(E_{1}-R_{33}\right) R_{21}^{(0)}+R_{23}^{(0)} R_{31}^{(0)}}
\end{aligned}
$$

а $R_{i j}^{0}$ определяются элементами $R_{i j}$ матрицы РГ преобразования (2.8), линеаризованного вблизи фиксированной точки $\left(r^{(0)}, u^{(0)}, \omega^{(0)}\right)$. Величина $V_{1}$, зависящая от большего собственного значения $E_{1}$ вышеупомянутой матрицы и ее элементов, приведена в работе [10]. Принимая во внимание значение температуры фазового перехода $\beta_{c} \widetilde{\Phi}(0)=$ 
1,1628 (случай $s=3, b=c$ ), находим $\tilde{c}_{1}^{(0)}=0,7630$. Отметим, что имеет место следующее равенство:

$$
s^{-\left(m_{\tau}+1\right)}=\tau^{\nu} c_{\nu}, \quad c_{\nu}=\left(\frac{\tilde{c_{1}}(0)}{\delta f_{0}}\right)^{\nu} .
$$

Критический показатель корреляционной длины $\nu$ определяется согласно известному соотношению [3]

$$
\nu=\frac{\ln s}{\ln E_{1}} .
$$

В случае использования модели $\rho^{6} \nu=0,640[5,9]$.

Величина $m_{\tau}$ характеризует размеры участка КР. Поскольку $m_{\tau} \sim \ln \tau$, то в пределе $\tau \rightarrow 0$ имеем $m_{\tau} \rightarrow \infty$ и тогда область КР сушествует для произвольно большого значения номера слоя $n$. При $\tau \neq 0$ выход из КР определяется величиной $\delta$ из (2.6). Для проведения расчетов удобно представить $\delta$ в виде

$$
\delta=\delta_{0} \tau^{\varkappa}
$$

где показатель $\varkappa \geq 0$. При $\varkappa>0$ выход из КР является малым и это позволяет описать поведение $r_{n}$ как функции $n$ при $\tau \rightarrow 0$. Для конечных значений $\tau$ величина $\delta$ порядка $r^{(0)}$ и здесь должен выполняться предельный переход $\varkappa \rightarrow 0$, что отвечает равенству $\delta=\delta_{0}$. Используя решения $\mathrm{PC}(2.7)$, запишем выражения для величин $r_{m_{\tau}+1}, u_{m_{\tau}+1}$, $\omega_{m_{\tau}+1}$. С учетом (2.14) имеем

$$
\begin{array}{ll}
r_{m_{\tau}+1}=-\beta \widetilde{\Phi}(0) \bar{r}_{m_{\tau}+1}, & \bar{r}_{m_{\tau}+1}=f_{0}\left(1-\delta_{0} \tau^{\varkappa}\right), \\
u_{m_{\tau}+1}=(\beta \widetilde{\Phi}(0))^{2} \bar{u}_{m_{\tau}+1}, & \bar{u}_{m_{\tau}+1}=\varphi_{0}\left(1+\frac{f_{0} \omega_{21}^{(0)}}{\sqrt{\varphi_{0}}} \delta_{0} \tau^{\varkappa}\right), \\
\omega_{m_{\tau}+1}=(\beta \widetilde{\Phi}(0))^{3} \bar{\omega}_{m_{\tau}+1}, & \bar{\omega}_{m_{\tau}+1}=\psi_{0}\left(1+\frac{f_{0} \varphi_{0} \omega_{31}^{(0)}}{\psi_{0}} \delta_{0} \tau^{\varkappa}\right) .
\end{array}
$$

В дальнейшем будем считать, что $\varkappa \ll 1$. Интегрирование статсуммы $(2.1)$ при $\tau>0$ с использованием негауссова распределения является необходимым только для отрицательных значений величины $r_{n}\left(\bar{r}_{n}>0\right)$. При $r_{n}>0\left(\bar{r}_{n}<0\right)$ распределение мод колебаний плотности спинового момента приобретает гауссов характер. Поведение $r_{n}$ с ростом номера слоя $n$ фазового пространства КП показано на рис. 1 . Как следует из результатов работ [2-4], при $n \leq m_{\tau}$ величина $r_{n}$ близка к $r^{(0)}$. При $n>m_{\tau}$ наблюдается рост $r_{n}$ с увеличением $n$. При некотором значении $n_{0}\left(n_{0}>m_{\tau}\right)$ коэффициент $r_{n}$ становится положительным и продолжает оставаться таким для всех $n>n_{0}$. Изменение знака величины $r_{n}$ достигается во втором слое интегрирования после точки выхода системы из КР при конкретном значении $\delta$. Далее все расчеты будем вести в $m_{\tau}+2$-м слое, а для удобства выберем $\delta_{0}=0,3$. Для второго слоя интегрирования $(m=2)$ относительно точки выхода на основе (2.7) запишем

$$
\begin{array}{ll}
r_{m_{\tau}+2}=-\beta \widetilde{\Phi}(0) \bar{r}_{m_{\tau}+2}, & \bar{r}_{m_{\tau}+2}=f_{0}\left(1-E_{1} \delta_{0} \tau^{\varkappa}\right), \\
u_{m_{\tau}+2}=(\beta \widetilde{\Phi}(0))^{2} \bar{u}_{m_{\tau}+2}, & \bar{u}_{m_{\tau}+2}=\varphi_{0}\left(1+\frac{f_{0} \omega_{21}^{(0)}}{\sqrt{\varphi_{0}}} E_{1} \delta_{0} \tau^{\varkappa}\right), \\
\omega_{m_{\tau}+2}=(\beta \widetilde{\Phi}(0))^{3} \bar{\omega}_{m_{\tau}+2}, & \bar{\omega}_{m_{\tau}+2}=\psi_{0}\left(1+\frac{f_{0} \varphi_{0} \omega_{31}^{(0)}}{\psi_{0}} E_{1} \delta_{0} \tau^{\varkappa}\right) .
\end{array}
$$




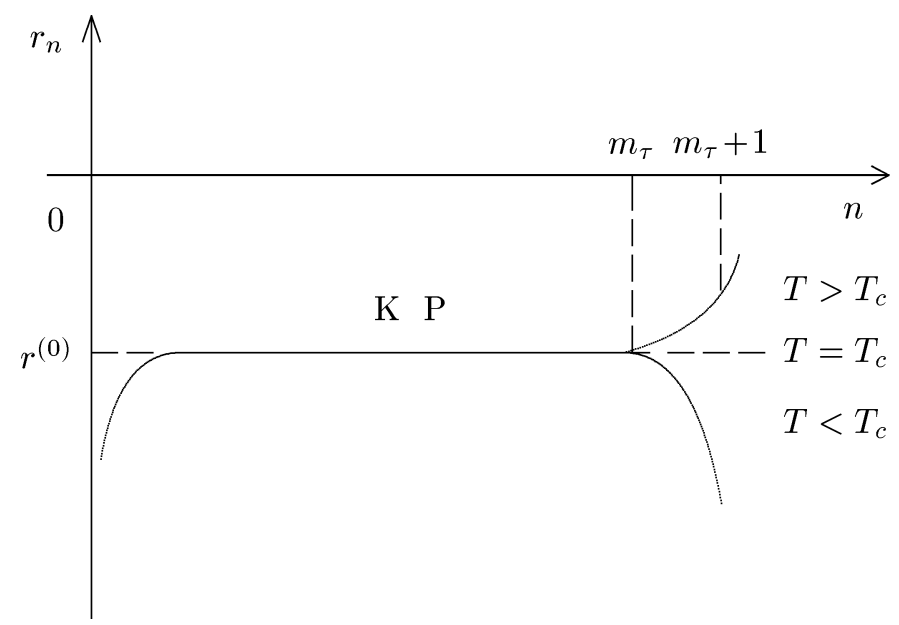

Рис. 1. Эволюция величины $r_{n}$ с ростом номера слоя $n$ фазового пространства КП.

График зависимости коэффициентов $\bar{r} \equiv \bar{r}_{m_{\tau}+m}, \bar{u} \equiv \bar{u}_{m_{\tau}+m}, \bar{\omega} \equiv \bar{\omega}_{m_{\tau}+m}$ от $\delta$ при $m=1$ приведен на рис. 2а, а при $m=2$ - на рис. $2 б$.

Точка выхода $m_{\tau}(2.10)$ системы из КР с учетом температурной зависимости $\delta(2.14)$ принимает форму

$$
m_{\tau}=-(1-\varkappa) \frac{\ln \tau}{\ln E_{1}}+m_{0}^{\prime}-1
$$

где $m_{0}^{\prime}$ определяется равенством для $m_{0}$ из $(2.11)$ при замене в нем $\delta$ величиной $\delta_{0}$. Принимая во внимание (2.14), находим

$$
s^{-\left(m_{\tau}+2\right)}=\tau^{(1-\varkappa) \nu} s^{-m_{0}^{\prime}-1} .
$$

Уравнение относительно параметра $\sigma$ получаем из $(2.4)$, где в качестве $E^{(1)}\left(\sigma, m_{\tau}+1\right)$ используется выражение $E^{(2)}\left(\sigma, m_{\tau}+2\right)$, включающее величины $r_{m_{\tau}+2}, u_{m_{\tau}+2}, \omega_{m_{\tau}+2}$. Используя (2.16), приходим к уравнению

$$
\begin{gathered}
\tau^{5 \nu / 2}\left[I_{11} \sigma_{0} \tau^{-2 \nu \varkappa}-I_{12} \sigma_{0} \tau^{-(2 \nu-1) \varkappa}-I_{21} \sigma_{0}^{3} \tau^{-\nu \varkappa}-\right. \\
\left.-I_{31} \sigma_{0}^{5}-I_{22} \sigma_{0}^{3} \tau^{(1-\nu) \varkappa}-I_{32} \sigma_{0}^{5} \tau^{\varkappa}\right]=-\beta h .
\end{gathered}
$$

Здесь введено следующее обозначение:

$$
\sigma=\sigma_{0} \tau^{\nu / 2}
$$



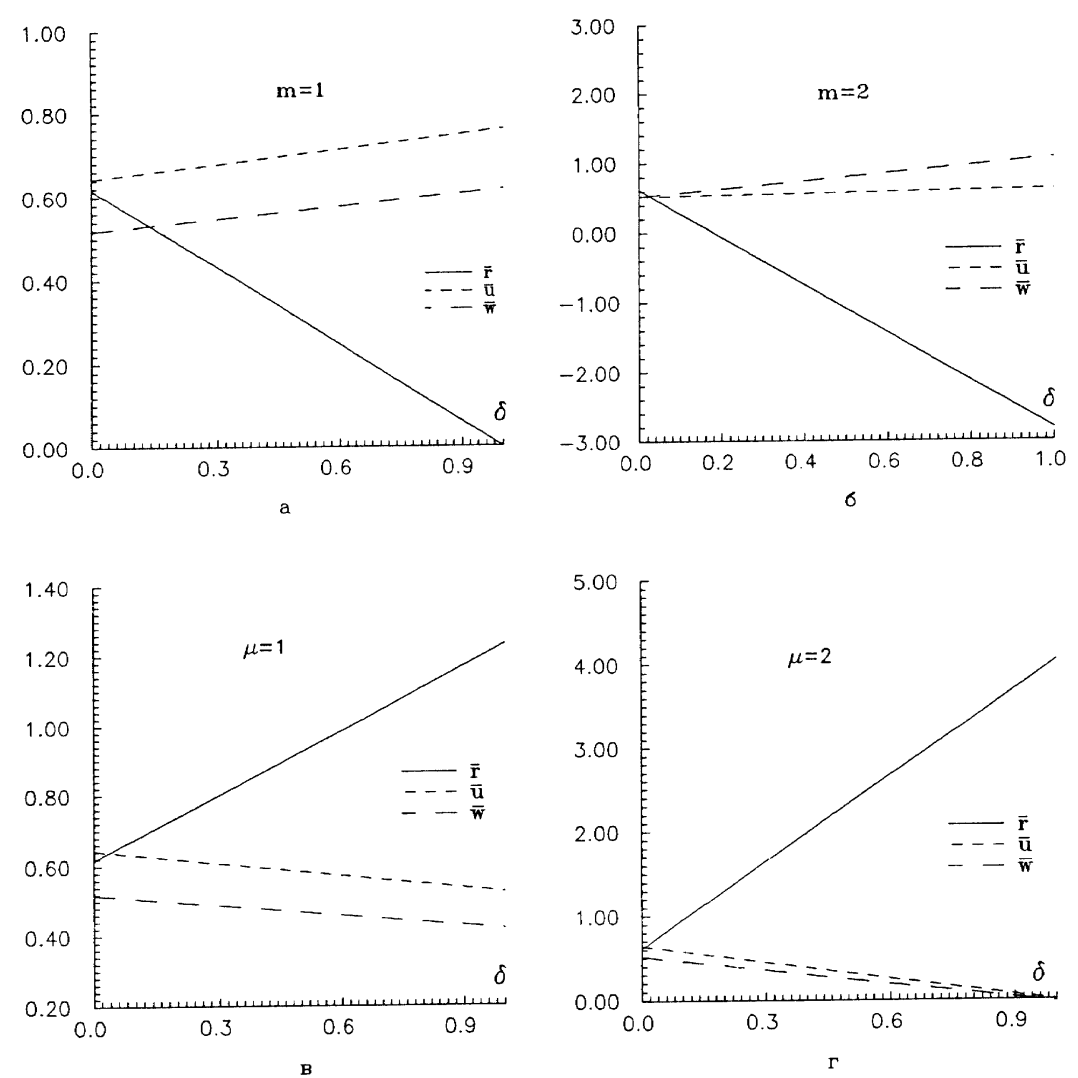

Рис. 2. График зависимости коэффициентов от $\delta$ в первом и во втором слоях интегрирования после выхода системы из КР: "а" и "б” при $\tau>0$; “в" и "г" при $\tau<0$.

Для коэффициентов $I_{m n}$ имеем

$$
\begin{aligned}
& I_{11}=f_{0} \beta \widetilde{\Phi}(0) s^{-2\left(m_{0}^{\prime}+1\right)}, \\
& I_{12}=I_{11} \delta_{0} E_{1}, \\
& I_{21}=\frac{\varphi_{0}}{6} s_{0}^{3}(\beta \widetilde{\Phi}(0))^{2} s^{-\left(m_{0}^{\prime}+1\right)}, \\
& I_{22}=I_{21} \delta_{0} \frac{f_{0}}{\sqrt{\varphi_{0}}} \omega_{21}^{(0)} E_{1}, \\
& I_{31}=\frac{\psi_{0}}{5 !} s_{0}^{6}(\beta \widetilde{\Phi}(0))^{3}, \\
& I_{32}=I_{31} \delta_{0} \frac{f_{0} \varphi_{0}}{\psi_{0}} \omega_{31}^{(0)} E_{1} .
\end{aligned}
$$

Слагаемые в левой части (2.19) пропорциональны различным степеням $\tau(\tau \ll 1)$. Два последних слагаемых являются членами высшего порядка малости по $\tau$, и ими можно 


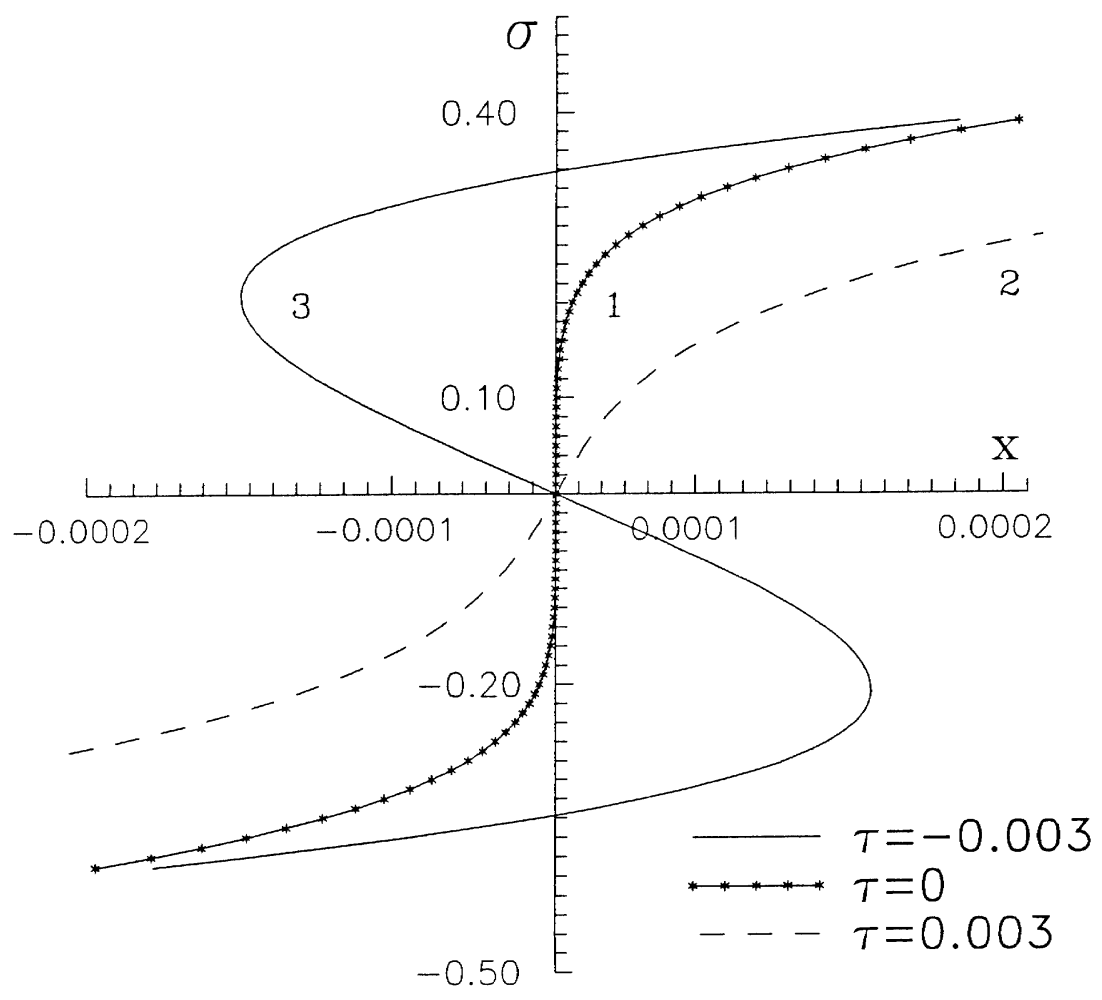

Рис. 3. Зависимость параметра порядка от внешнего поля.

пренебречь. Поэтому уравнение (2.19) перепишем в виде

$$
\beta h=\left[I_{31} \sigma_{0}^{5}+I_{21} \sigma_{0}^{3} \tau^{-\nu \varkappa}+I_{12} \sigma_{0} \tau^{-(2 \nu-1) \varkappa}-I_{11} \sigma_{0} \tau^{-2 \nu \varkappa}\right] \tau^{5 \nu / 2} .
$$

В пределе $\varkappa \rightarrow 0$ находим

$$
I_{31} \sigma^{5}+I_{21} \sigma^{3} \tau^{\nu}+\left(I_{12}-I_{11}\right) \sigma \tau^{2 \nu}=\beta h
$$

Величины $I_{m n}$ определены в (2.21). Уравнение (2.23) является уравнением состояния при $T>T_{c}$. Из него можно получить дополнительное условие для величины $\delta_{0}$, входяшей в $\delta$ (см. (2.6), (2.14)). Принимая во внимание положительность коэффициента возле $\sigma^{2}$ в (2.5) или коэффициента при $\sigma$ в $(2.23)$, имеем

$$
\begin{gathered}
I_{12}-I_{11}=I_{11}\left(-1+\delta_{0} E_{1}\right), \\
\delta_{0} E_{1}>1, \quad \delta_{0}>\delta_{0}^{\prime}=1 / E_{1}, \quad \delta_{0}^{\prime}=0,1799 .
\end{gathered}
$$




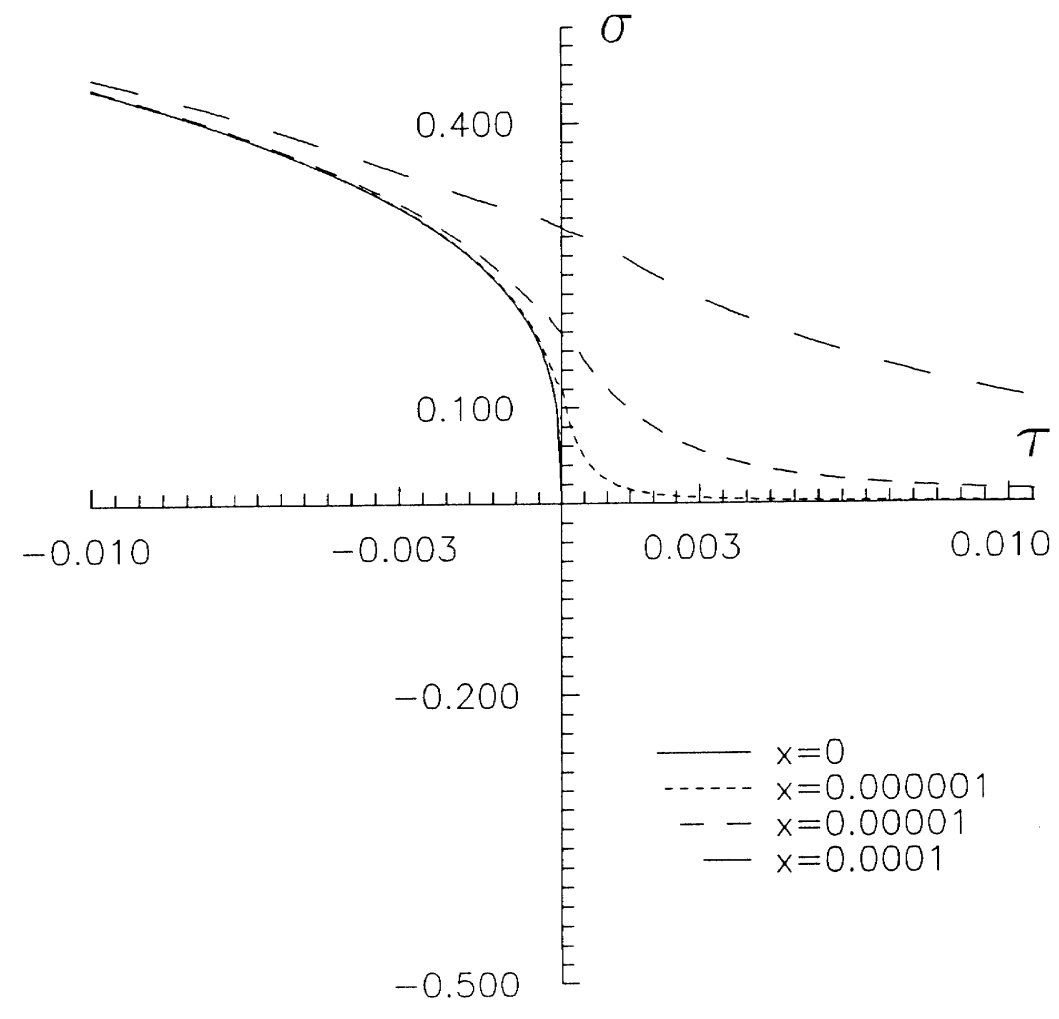

Рис. 4. Температурная зависимость параметра порядка однокомпонентной спиновой системы в рамках модели $\rho^{6}$ при $b=c(2 \sqrt{3})^{-1}$ и $s=3$.

Расчет уравнения состояния в области температур $T<T_{c}$ осушествим аналогично описанной выше схеме. Определим точку выхода системы из КР при $\tau<0$. Как следует из рис. 1 , для этого температурного диапазона имеем

$$
\frac{r_{\mu_{\tau}+1}-r^{(0)}}{r^{(0)}}=\delta .
$$

Учитьвая выражение $r_{n}(2.7)$, из (2.25) получаем уравнения для $\mu_{\tau}$ :

$$
c_{1} E_{1}^{\mu_{\tau}+1}=-\delta f_{0} \beta \widetilde{\Phi}(0) .
$$

Величину $\delta$ представим в виде

$$
\delta=\delta_{0}|\tau|^{\varkappa} .
$$

Точка выхода из $\mathrm{KP}$ при $T<T_{c}$ описывается соотношением

$$
\mu_{\tau}=-(1-\varkappa) \frac{\ln |\tau|}{\ln E_{1}}+m_{0}^{\prime}-1 .
$$




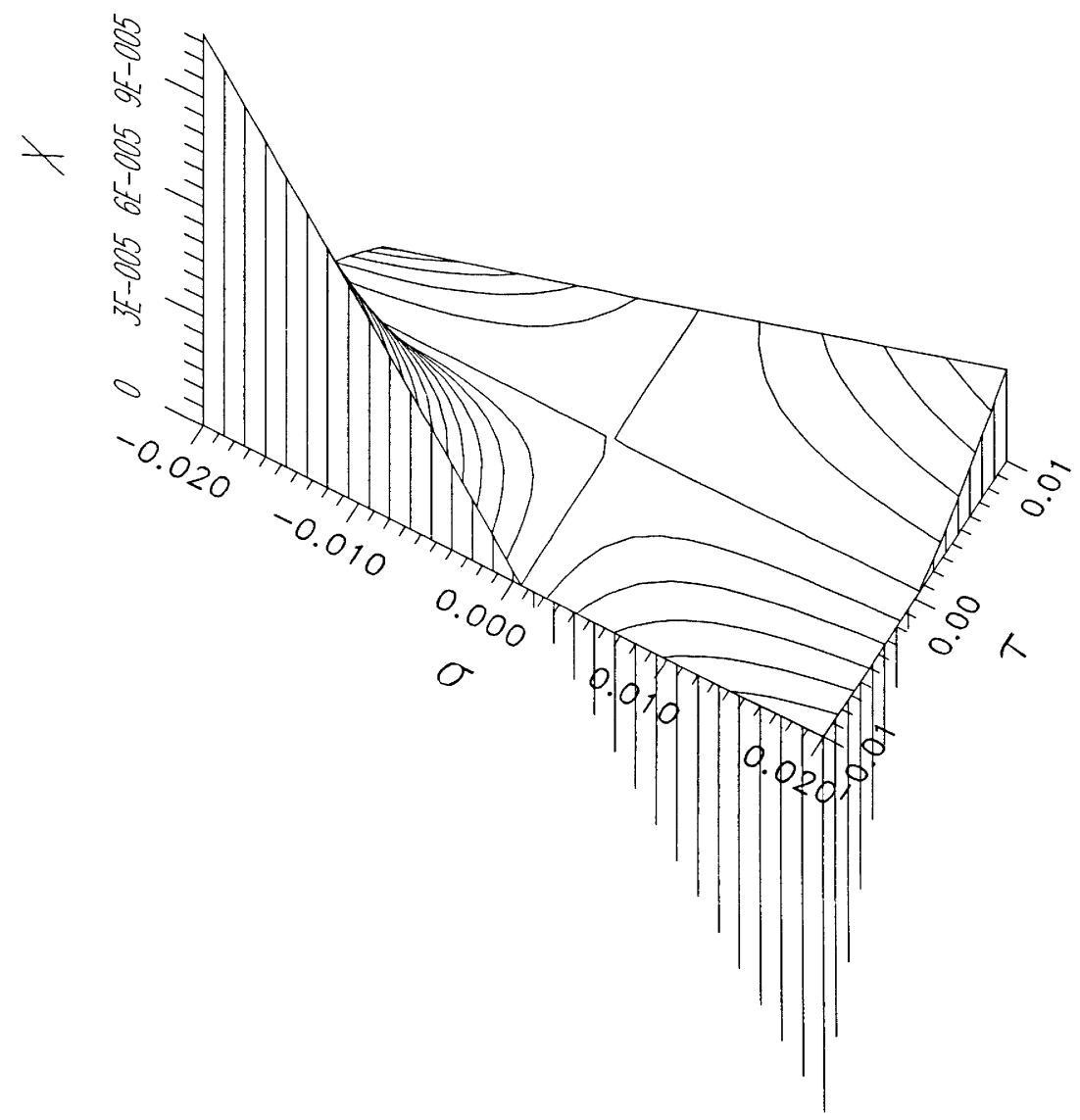

Рис. 5. Параметр порядка в непосредственной окрестности критической температуры при соответствии параметров экспоненциально убываюшего потенциала взаимодействию ближайших соседей.

Исходя из $(2.7),(2.26)$ и $(2.27)$ находим коэффициенты шестерной плотности меры $\mu_{\tau}+$ 2-й блочной структуры:

$$
\begin{aligned}
& r_{\mu_{\tau}+2}=-\beta \widetilde{\Phi}(0) \bar{r}_{\mu_{\tau}+2}, \quad \bar{r}_{\mu_{\tau}+2}=f_{0}\left(1+E_{1} \delta_{0}|\tau|^{\varkappa}\right), \\
& u_{\mu_{\tau}+2}=(\beta \widetilde{\Phi}(0))^{2} \bar{u}_{\mu_{\tau}+2}, \quad \bar{u}_{\mu_{\tau}+2}=\varphi_{0}\left(1-\frac{f_{0} \omega_{21}^{(0)}}{\sqrt{\varphi_{0}}} E_{1} \delta_{0}|\tau|^{\varkappa}\right), \\
& \omega_{\mu_{\tau}+2}=(\beta \widetilde{\Phi}(0))^{3} \bar{w}_{\mu_{\tau}+2}, \quad \bar{w}_{\mu_{\tau}+2}=\psi_{0}\left(1-\frac{f_{0} \varphi_{0} \omega_{31}^{(0)}}{\psi_{0}} E_{1} \delta_{0}|\tau|^{\varkappa}\right) .
\end{aligned}
$$

Эволюция величин $\bar{r} \equiv \bar{r}_{\mu_{\tau}+\mu}, \quad \bar{u} \equiv \bar{u}_{\mu_{\tau}+\mu}, \bar{\omega} \equiv \bar{\omega}_{\mu_{\tau}+\mu}$ с ростом $\delta$ в первом $(\mu=1)$ и во втором слоях $(\mu=2)$ интегрирования после выхода системы из $\mathrm{KP}$ при $\tau<0$ изображена соответственно на рис. 2в и г. Проведя необходимые вычисления, запишем 
уравнение для $\sigma$

$$
\begin{aligned}
\sigma f_{0} & \beta \widetilde{\Phi}(0) s^{-2\left(m_{0}^{\prime}+1\right)}|\tau|^{2 \nu(1-\varkappa)}+\sigma f_{0} \beta \widetilde{\Phi}(0) \delta_{0} E_{1}|\tau|^{\varkappa} s^{-2\left(m_{0}^{\prime}+1\right)}|\tau|^{2 \nu(1-\varkappa)}- \\
& -\frac{\sigma^{3}}{6} s_{0}^{3} \varphi_{0}(\beta \widetilde{\Phi}(0))^{2} s^{-\left(m_{0}^{\prime}+1\right)}|\tau|^{\nu(1-\varkappa)}+ \\
& +\frac{\sigma^{3}}{6} s_{0}^{3} \sqrt{\varphi_{0}}(\beta \widetilde{\Phi}(0))^{2} s^{-\left(m_{0}^{\prime}+1\right)} \delta_{0} f_{0} \omega_{21}^{(0)} E_{1}|\tau|^{\nu(1-\varkappa)}|\tau|^{\varkappa}- \\
& -\frac{\sigma^{5}}{5 !} s_{0}^{6} \psi_{0}(\beta \widetilde{\Phi}(0))^{3}+\frac{\sigma^{5}}{5 !} s_{0}^{6}(\beta \widetilde{\Phi}(0))^{3} \delta_{0} f_{0} \varphi_{0} \omega_{31}^{(0)} E_{1}|\tau|^{\varkappa}+\beta h=0 .
\end{aligned}
$$

Осушествим в (2.30) замену переменных

$$
\sigma=\sigma_{0}|\tau|^{\nu / 2}
$$

Получаем

$$
\begin{gathered}
|\tau|^{5 \nu / 2}\left[I_{11} \sigma_{0}|\tau|^{-2 \nu \varkappa}+I_{12} \sigma_{0}|\tau|^{-(2 \nu-1) \varkappa}-I_{21} \sigma_{0}^{3}|\tau|^{-\nu \varkappa}-\right. \\
\left.-I_{31} \sigma_{0}^{5}+I_{22} \sigma_{0}^{3}|\tau|^{(1-\nu) \varkappa}+I_{32} \sigma_{0}^{5}|\tau|^{\varkappa}\right]=-\beta h .
\end{gathered}
$$

В пределе $\varkappa \rightarrow 0$ приходим к уравнению

$$
I_{31} \sigma^{5}+I_{21} \sigma^{3}|\tau|^{\nu}-\left(I_{11}+I_{12}\right) \sigma|\tau|^{2 \nu}=\beta h
$$

\section{3. ТЕМПЕРАТУРНАЯ И ПОЛЕВАЯ ЗАВИСИМОСТЬ ПАРАМЕТРА ПОРЯДКА ТРЕХМЕРНОЙ МОДЕЛИ ИЗИНГА}

Запишем уравнения (2.23) и (2.33) в более удобной форме. Находим

$$
D \sigma^{5}+G \tau^{\nu} \sigma^{3}+B\left(\delta_{0} E_{1}-1\right) \tau^{2 \nu} \sigma-\beta h=0
$$

для $\tau>0$ и

$$
D \sigma^{5}+G|\tau|^{\nu} \sigma^{3}-B\left(\delta_{0} E_{1}+1\right)|\tau|^{2 \nu} \sigma-\beta h=0
$$

для $\tau<0$. Здесь

$$
\begin{aligned}
D & =(\beta \widetilde{\Phi}(0))^{3} s_{0}^{6} \frac{\psi_{0}}{5 !}, \\
G & =\frac{(\beta \widetilde{\Phi}(0))^{2}}{6} s_{0}^{3} s^{-1} \varphi_{0} c_{\nu}^{\prime}, \\
B & =\beta \widetilde{\Phi}(0) f_{0} s^{-2}\left(c_{\nu}^{\prime}\right)^{2} .
\end{aligned}
$$

Выражение для $c_{\nu}^{\prime}$ получаем из $c_{\nu}(2.12)$, положив $\delta=\delta_{0}$. В обшем случае уравнения (3.1) и (3.2) можно представить одним, а именно:

$$
D \sigma^{5}+G|\tau|^{\nu} \sigma^{3}+B\left(\delta_{0} E_{1} \frac{\tau}{|\tau|}-1\right)|\tau|^{2 \nu} \sigma-\beta h=0
$$

Решения данного уравнения являются функциями температуры, внешнего поля и микроскопических параметров системы:

$$
\sigma=\sigma(\tau, x, b / c)
$$




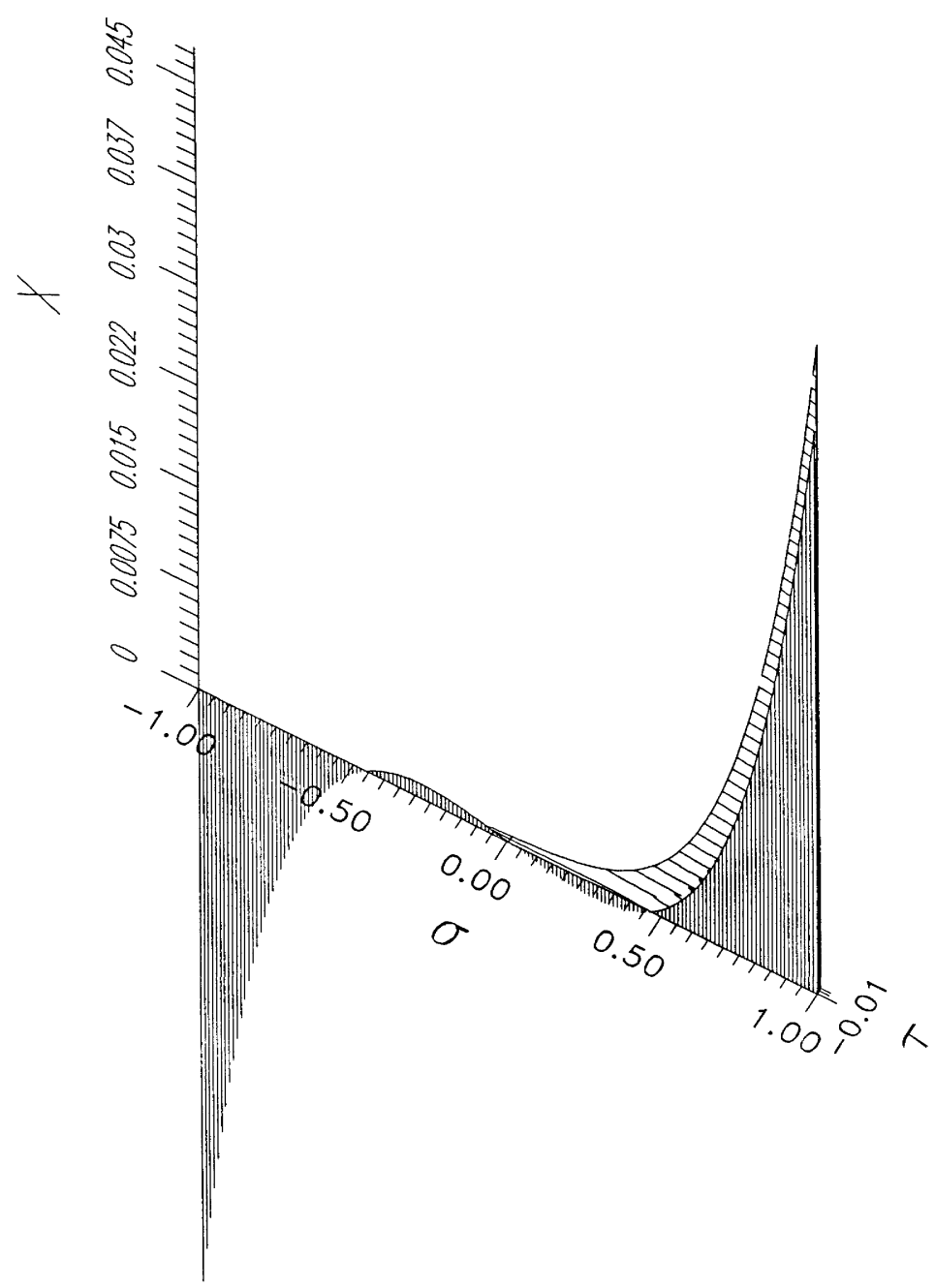

Рис. 6. Поведение параметра порядка во внешнем поле.

где $x=\beta h-$ обобщенное поле.

В случае $b=c(2 \sqrt{3})^{-1}$ имеем

$$
D=0,0302, \quad G=0,2522, \quad B=0,4886 .
$$

Для простоты расчетов ограничимся этим частным случаем и рассмотрим зависимость параметра порядка (3.5) от температуры и поля. Подставим в уравнение $(3.4) \tau=0$. В результате исчезают слагаемые, пропорциональные $|\tau|^{\nu},|\tau|^{2 \nu}$ и

$$
\sigma^{5}=\frac{x}{D}
$$

Отсюда определяем критический показатель $\delta$, который характеризует зависимость $\sigma=$ $\sigma_{\mathrm{Kp}} x^{1 / \delta}$ :

$$
\delta=5 \text {. }
$$




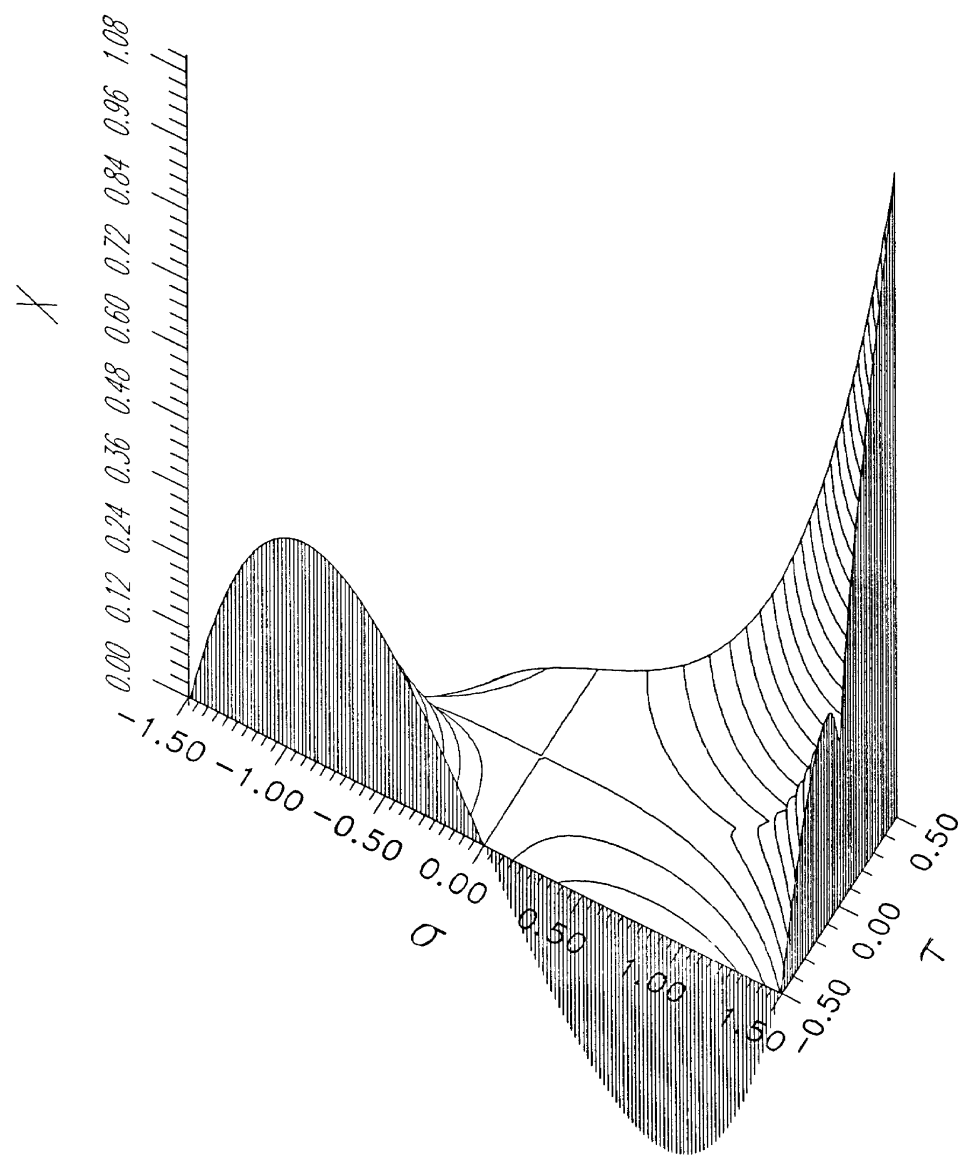

Рис. 7. Температурная и полевая зависимости решения $\sigma=\sigma(\tau, x)$ уравнения состояния трехмерной модели Изинга.

Критическая амплитуда

$$
\delta_{\mathrm{Kp}}=(D)^{1 / 5} \text {. }
$$

Зависимость $\sigma=\sigma(x)$ для $b=c(2 \sqrt{3})^{-1}$ и $\tau=0$ изображена на рис. 3 кривой 1 . Для температур $\tau>0$ кривые $\sigma=\sigma(x)$ находятся между кривой 1 и осью $0 x$ (см. кривую 2 ). При температурах $\tau<0$ такая зависимость представлена кривой $3(\tau=-0,003)$. х В случае нулевого внешнего магнитного поля $(x=0)$ уравнение состояния $(3.4)$ имеет вид

$$
D \sigma^{5}+G|\tau|^{\nu} \sigma^{3}+B\left(\delta_{0} E_{1} \frac{\tau}{|\tau|}-1\right)|\tau|^{2 \nu} \sigma=0
$$

Оно имеет тривиальное решение

$$
\sigma=0
$$

и решения типа

$$
\sigma^{2}=\left\{-\frac{G}{2 D} \pm\left[\frac{G^{2}}{4 D^{2}}-\frac{B}{D}\left(\delta_{0} E_{1} \frac{\tau}{|\tau|}-1\right)\right]^{1 / 2}\right\}|\tau|^{\nu}
$$

5 Теоретическая и математическая физика, т. 107, № 2, 1996 г. 
Знак плюс перед квадратной скобкой в правой части равенства (3.12) соответствует физическим решениям. Легко видеть, что при $T>T_{c}$ действительных решений нет, а при $T<T_{c}$ оба решения действительны. Знак минус связан с мнимыми решениями. Заметим, что при получении уравнения состояния в настоящей работе не учитывались вклады, определяюшие поправки к скейлингу. Они пропорциональны малой (в силу малости $\tau)$ величине $|\tau|^{\Delta_{l}}$, где

$$
\Delta_{l}=-\frac{\ln E_{l}}{\ln E_{1}}, \quad l=2,3 .
$$

Для $b=c$ и параметра $s=3$ разбивки фазового пространства КП на слои в приближении модели $\rho^{6}$ (см. [9]) следующие:

$$
\Delta_{1}=0,503, \quad \Delta_{2}=3,379 .
$$

Кривые зависимости $\sigma=\sigma(\tau)$ при определенных значениях $x$ (или $h$ ) приведены на рис. 4. В общем случае $\sigma=\sigma(\tau, x)$ имеет вид поверхности, которая приведена на серии рис. $5-7$. На первом из них (рис. 5) имеем близкую к критической область температур $-0,01<\tau<0,01$. Однако здесь не видно действительной зависимости $\sigma$ от $h$. Это хорошо видно на рис. 6 , где увеличен интервал значений величины $\sigma$. Более обшее поведение поверхности $\sigma=\sigma(\tau, x)$ представлено на рис. 7 .

\section{Список литературы}

[1] Юхновский И.Р. // ТМФ. 1978. Т. 36. №3. С. 373-399.

[2] Юхновский И.Р. Фазовые переходы второго рода. Метод коллективных переменных. Киев: Наукова думка, 1985.

[3] Козловский М.П., Пылюк И.В., Юхновский И.Р. // ТМФ. 1991. Т. 87. № 2. С. 293-316.

[4] Козловский М.П., Пылюк И.В., Юхновский И.Р. // ТМФ. 1991. Т. 87. № 3. С. 434-455.

[5] Козловский М.П. // ТМФ. 1989. Т. 78. № 3. С. 422-433.

[6] Ландау Л.Д., Лифиич Е.М. Статистическая физика. Ч. І. М.: Наука, 1976.

[7] Изюмов Ю.А., Сыромятников В.Н. Фазовые переходы и симметрия кристаллов. М.: Наука, 1984.

[8] Le Guillou J.C., Zinn-Justin J. // Phys. Rev. B. 1980. V. 21. № 9. P. 3976-3998.

[9] Козловский М.П., Пълюк И.В. Термодинамика трехмерного изинговского ферромагнетика в окрестности точки фазового перехода в рамках модели $\rho^{6}$. Сравнение с моделью $\rho^{4}:$ Препринт ИТФ-90-81Р. Киев: Институт теоретической физики, 1990.

[10] Козловский М.П., Пьлюк И.В. Расчет термодинамических функций вблизи точки фазового перехода в приближении шестерной базисной меры: Препринт ИТФ-87-9Р. Киев: Институт теоретической физики, 1987.

Институт физики конденсированных систем

Национальной академии наук Украины

\section{V.V. Dukhovii, M. P. Kozlovskii, I. V. Pylyuk EQUATION OF STATE IN 3D ISING MODEL FROM MICROSCOPIC LEVEL CALCULATION}

A new method for derivating of the equation of state in the $3 \mathrm{~d}$ Ising model on the simple cubic lattice with the exponentially decreasing potential is proposed. This equation describes the order parameter of the system as a function of the temperature, external field and the microscopic parameters of the system in the critical region. Numerical investigation of this function is performed for the case when the potential parameters correspond to the nearest neighbours interaction. 